\title{
A GROUND CONTROL STATION FOR THE UAV FLIGHT SIMULATOR
}

\author{
Sławomir ROMANIUK*, Zdzisław GOSIEWSKI*, Leszek AMBROZIAK* \\ *Department of Automatic Control and Robotics, Faculty of Mechanical Engineering, Bialystok University of Technology, \\ ul. Wiejska 45C, 15-351 Białystok, Poland \\ s.romaniuk@doktoranci.pb.edu.pl, z.gosiewski@pb.edu.pl, l.ambroziak@pb.edu.pl
}

received 13 July 2015, revised 3 February 2016, accepted 8 February 2016

\begin{abstract}
In the paper implementation of a ground control station for UAV flight simulator is shown. The ground control station software is in cooperation with flight simulator, displaying various aircraft flight parameters. The software is programmed in $\mathrm{C}++$ language and utilizes the windows forms for implementing graphical content. One of the main aims of the design of the application was to simplify the interface, simultaneously maintaining the functionality and the eligibility. A mission can be planned and monitored using the implemented map control supported by waypoint list.
\end{abstract}

Key words: Ground Control Station, Flight Simulator

\section{INTRODUCTION}

Having miniaturized wide scope of measurement and communication devices and reduced costs of propelling devices, relatively cheap Unmanned Aerial Vehicles (UAVs) development has intensified. Trend of utilizing autopilot's capabilities for performing different applications of UAVs is arising. Contemporary autopilot systems are able to stabilize object's rotary motion along all three axes corresponding to pitch, roll and yaw angles. Those basic abilities allows implementing more complicated routines such as autonomous starting and landing, navigation through desired waypoints, circulation around given point. Furthermore unmanned aerial vehicles may be equipped with many different measurement devices, cameras, and miscellaneous sensors. Hence by the aggregation of mentioned elements it is possible to plan different UAV's missions for many modern appliances e.g. searching for lost. Although a number of adoptions is possible, there is additional necessity for some kind of supplementary system which would give final user the eventuality of planning, monitoring and controlling the UAV. This system may be represented by ground control station (GCS).

Ground control station may have many different capabilities, however there are few that are usually implemented. One of the requirement that GCS is usually bound to fulfill is acquiring and displaying data relating UAV flight parameters, which may be showed as text or visualized through different graphical elements. Furthermore ground control station should have the possibility of steering the UAV behavior by sending appropriate configuration and control commands to it.

As the one of the main components of any Unmanned Aerial System (UAS), ground control station serves as the device, the user can monitor and control UAV through. Hence it has to be as intuitive and clear as possible, to decrease the overall operator workload and improve operational parameters such as reaction time of the operator.

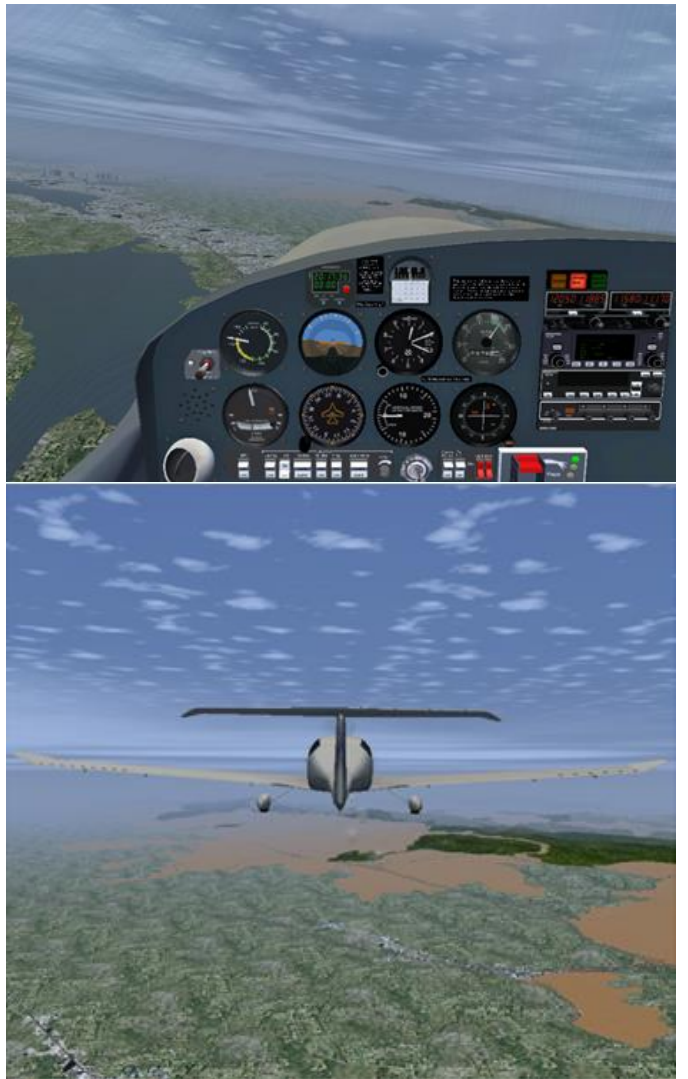

Fig. 1. The "FlightGear" application (Zachary et al., 2013)

There is unextinguished work to increase the functionality of ground control station, since they are reliable for successful mission accomplishment of unmanned aerial vehicles. Hence the adoption of multimodality has occurred in order to improve the interaction quality between the operator and GCS. Elements like text-to-speech synthesis, voice recognition, three dimensional 
projection may be useful in decreasing the operators workload and effort. Nevertheless addition of such communication channels should be properly designed, otherwise it can redound to undesirable overload of incoming information (Perez et al., 2013).

There are commercial ground control stations like the one by Airware (http://www.airware.com/). Likewise they are quite a few open-source projects like OpenPilot Ground Control Station (http: //www.openpilot.org/) or the QGroundControl (http://www.qground control.org/). However usage of aforementioned ground control station projects has disadvantages. One of the problem that arises, involves adapting them into system performing assumed tasks.

The direct implementation of ground control station for chosen autopilot system would be time demanding, awkward and weather dependent, since programming of such software requires constant interaction with target device. Furthermore many changes would have to be verified during the flight, causing additional inconveniences. Hence ground control station depicted hereafter is based on the flight simulator. This approach excludes the need of using communication devices, because both, the ground control station and the flight simulator may run on the same computer. Moreover by means of using flight simulator it is possible to achieve aerial situations that are dangerous, hard to perform or even unreachable with real objects.

\section{SYSTEM ARCHITECTURE}

The flight simulator software, a ground control station software and the computer those programs run on, are the elements of the validation system.

FlightGear (http://www.flightgear.org/) is the program used as the flight simulator component. It is an open-source program, that has different versions supporting many popular platforms (Windows, Linux, Mac). FlightGear was created as the simulation tool that can be used as the pilot training software, industry engineering tool. Nevertheless it can be used in the academic applications due to its implementation of many different aircrafts dynamic models. Furthermore FlightGear allows communication with individual software programs as well as external devices through network socket connection. This simulator allows previewing the flight from both inside and outside of the aircraft (Fig. 1). Moreover adverse weather effects such as wind may be simulated, giving the potentiality of aircraft's behavior assessment in the extreme conditions (http://www.flightgear.org/).

Ground control station is basing on the data acquired from the flight simulator. These programs are utilizing the TCP in order to communicate each other. The ground control station starts first before the flight simulator for the creation of a server. In the next step the starting flight simulator connects to the previously created server. Followed by the connection of the GCS to the flight simulator. When the connection is successfully established, FlightGear sends output data in the predefined form. It can output many various parameters ranging from the position and orientation ones through gear and onboard systems states, engines speeds, control surface positions ending with velocities and accelerations. Parameters that are demanded at the output may be arbitrarily adjusted according to the current needs, by means of the configuration file. Moreover there is possibility of modifying the frequency at which the program actualizes the output. It was necessary to implement adequate class, which is responsible for TCP connection and packets recognition for data acquisition.

\subsection{Transmission Control Protocol}

Transmission Control Protocol (TCP) is the transport layer protocol. TCP is responsible for the correctness of transmitted data. It is situated just above a basic Internet Protocol (IP). TCP utilizes IP for sending and receiving variable-length segments of information. Furthermore internet protocol provides ability of reassembling fragmented TCP segments, assuring delivery through multiple networks (http://www.tools.ietf.org/html/rfc793, http://www.students.mimuw.edu.pl/SO/Linux). In the following work TCP is used to maintain communication between the application of ground control station and the flight simulator.

\section{GROUND CONTROL STATION DESIGN}

The ground control station was designed in order to improve operator's awareness of UAV's current situation. Moreover the system was aimed to be operated by a single user, enabling him to control UAV missions with little effort. The overall design of the graphical interface had to be kept on the simple form. It was achieved by placing constituent elements in the organized form, which simultaneously display as well as visualize a set of important flight parameters.

In the proposed design of ground control station, many different elements presenting basic flight parameters are included. Some of these parameters are visualized, in order to increase their eligibility, while the rest of them are displayed in the form of numbers.

The ground control station software was programmed in the $\mathrm{C}++$ language utilizing the standard WinApi libraries. This approach gave possibility of running the mentioned program on Windows class systems. Well documented WinApi libraries allow convenient development of various application providing access to many basic system resources including file systems, devices, network services, graphical user interfaces, error handling (http://msdn.microsoft.com/en-us/library). However the graphical content of WinApi provides functions for drawing some basic geometric figures, hence there was necessity of writing own classes for creating and updating controls that visualize current orientation and position of the monitored aircraft.

Ground control station window is divided into two main areas, each of which contains a set of different controls.

\subsection{Aircraft area}

Aircraft area that is located at the left side of GCS window presents some basic information of the aircraft's current orientation and position. Attitude of the object is presented in the three consequent indicators that visualize pitch, roll and yaw respectively. Pitch and roll indicators look similarly. They consist out of an circle inside of which aircraft's graphical visualization is presented. At the outside of that circle, short lines with adequate numbers are placed in order to preview the aircraft's inclination. Numbers are placed in the way that their center of symmetry covers the center of the previously mentioned circle. 


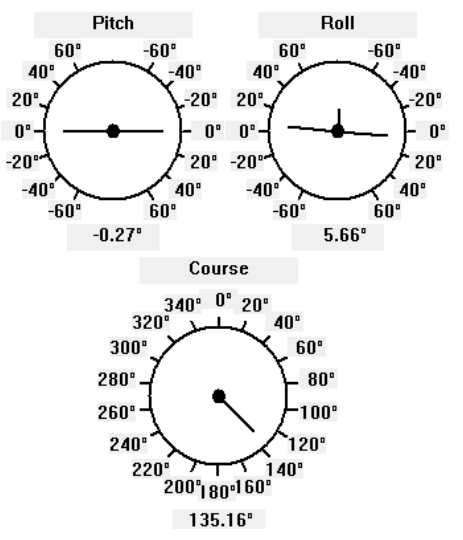

Fig. 2. Pitch, roll and yaw indicators

That approach increases the user awareness of the current aircraft orientation, by enabling him indicating the pitch and roll angles from both sides respectively. At the bottom of each indicator the field displaying amending value of pitch and roll angles respectively is placed. The general yaw indicator outlook is almost the same as pitch and roll ones except that, the numbers indicating the course angle are ranged from $0^{\circ}$ up to $360^{\circ}$.

In the next section of the aircraft's area, parameters of the aircrafts orientation and position are displayed in the form of list.

At the first, pitch, roll and yaw angles are presented. They are displayed both in the degrees and in the radians, however angles with degrees units are slightly less precisely presented than in the foregoing indicators. Subsequently the velocity values are displayed both in the $\mathrm{km} / \mathrm{h}$ and in the $\mathrm{m} / \mathrm{s}$ units. Values of latitude, longitude and height are displayed at the end of the list alternately. Latitude and longitude are shown in two forms: degrees only one and degrees, minutes, seconds alternative one.

\begin{tabular}{|l|c|c|c|} 
Parameters & & & \\
Pitch: & $-0.3^{\circ}$ & $->$ & $-0.00 \mathrm{rad}$ \\
\hline Roll: & $5.7^{\circ}$ & $->$ & $0.10 \mathrm{rad}$ \\
\hline Yaw: & $135.2^{\circ}$ & $->$ & $2.36 \mathrm{rad}$ \\
Velocity: & $276.1 \mathrm{~km} / \mathrm{h}$ & $->$ & $76.7 \mathrm{~m} / \mathrm{s}$ \\
\hline Latitude: & $37.71488^{\circ}$ & $->$ & $37^{\circ} 42^{\prime} 53^{\prime \prime}$ \\
\hline Longitude: & $-122.31856^{\circ}$ & $->$ & $-122^{\circ}-19^{\prime}-6^{\prime \prime}$ \\
\hline Height: & $1151.6 \mathrm{~m}$ & & \\
\hline
\end{tabular}

Fig. 3. Aircraft's flight parameters section

Afterwards values of elevator, ailerons, rudder and flaps position are presented in the last section of the aircraft area. These values are ranged from $-100 \%$ to $100 \%$ and are representing maximum deflection in opposite directions. Number of digits that are displayed may be adjusted in order to increase or decrease the precision.

\begin{tabular}{|l|c|}
\hline Control surface positions & \\
\hline Elevator: & $6.6 \%$ \\
Ailerons: & $27.6 \%$ \\
Rudder: & $-3.6 \%$ \\
Flaps: & $0.0 \%$ \\
\hline
\end{tabular}

Fig. 4. Aircraft's control surface positions section

\subsection{Map area}

Map area contains, as the name suggests, the visualized waypoints, but it is not the only one element displayed. Additionally this area includes the list of waypoints, three fields to type in new coordinates and a button for adding a new waypoint.

On the edges of the map, latitude and longitude in the degrees form is presented respectively at the left side and at the bottom of the map. On opposite sides of the map, those parameters represented in the meters units are shown. Map is displaying two types of points. First of them is black thick circle with a black short line. Those show current position and course of the aircraft. The other type of point is a red thin circle. A set of this points represents the position of inflicted waypoints. While the aircraft moves, it leaves a black thick line on the map which is drew to indicate aircraft's travelled path.

The map visualization allows planning aircrafts route. Moreover it is possible to add new waypoints by right-clicking on the map, which is an alternative to adding the waypoints by typing their coordinates in.

Below the map list of existing waypoints is presented. List has elements that consist of latitude, longitude and height values, given in degrees and meters units respectively, separated by comma character. List of the waypoints is aided with three fields that allow specifying latitude, longitude and height of newly created waypoint, and the button that is used to confirm addition of the new waypoint. New waypoint is placed at the end of the list of waypoints.

All the waypoints are managed by the autopilot. Application of the ground control station sends requests, with coordinates, to the autopilot in order to add new waypoint.

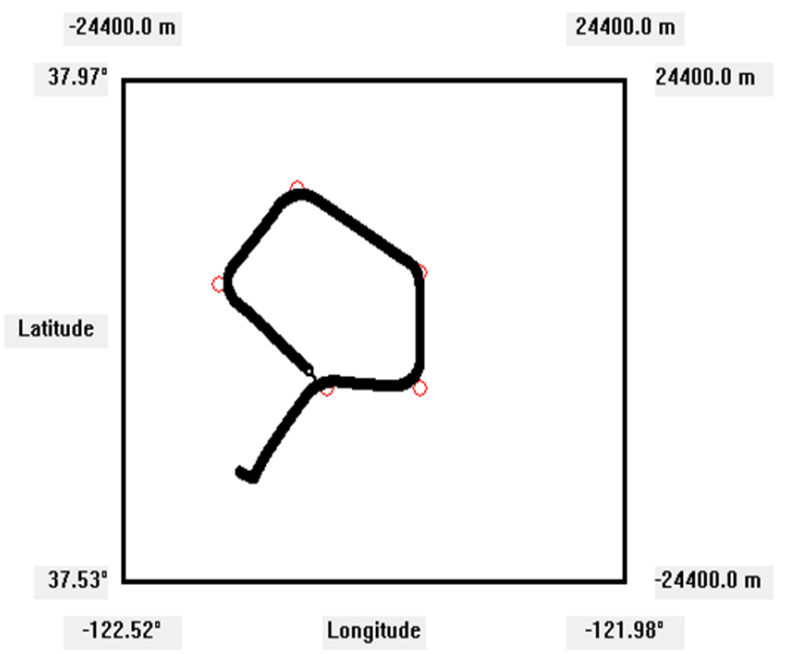

Fig. 5. Ground control station's map

In the navigation section parameters according control of the UAV route are presented. An alpha angle is the angle to the previous waypoint from the current one, whilst the beta angle is the angle to the next waypoint determined from the current waypoint. Gamma angle is the bisector of the alpha and beta angles. Phi angle is the sum of two angles. First one is the value calculated from the characteristic point placed on the bisector to the current position of aircraft, while the other one is $\pi / 2$. Distance is determined from the current aircraft position to the current waypoint. 


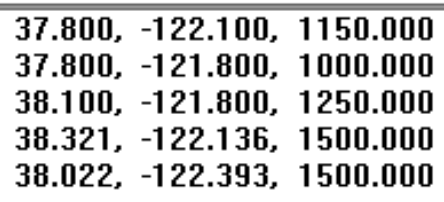

$37.800,-121.800,1000.000$

$38.100,-121.800,1250.000$

$38.321,-122.136,1500.000$

38.022, $-122.393,1500.000$

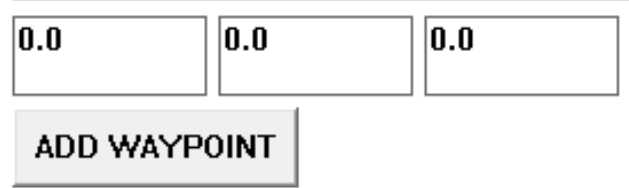

Fig. 6. List of the set waypoints with controls for adding new one

Presented GCS has the possibility of logging achieved data into file. Basing on the information from this file it is possible to further analyze, post-process the flight parameters of the aircraft.

\section{GROUND CONTROL STATION TESTING}

In order to examine and present the capabilities of designed GCS, test flight has been performed. The chosen aircraft model was Diamond DA40, which model can be adopted as the UAV platform. It is an $8.1 \mathrm{~m}$ long, $1.98 \mathrm{~m}$ high civil aircraft which can cruise at the speeds of up to $279 \mathrm{~km} / \mathrm{h}$, and has range of around $1341 \mathrm{~km}$ and wingspan of $11.9 \mathrm{~m}$ (http://www.diamondaircraft. com/).

The aircraft has flew along the arranged route, which was defined by means of waypoints. Preview of the preset waypoints is presented on the map while their coordinates are detailed in the waypoint list. Three of the waypoints were predefined in the software, while the other were added using the ground control station interface. First two were inserted utilizing the ability of map right click addition. Rest of them were configured by typing in their coordinates in three corresponding fields rightly and confirming with adequate button. Having configured the path, "START" button was pressed to commence the flight.

When the aircraft started it became apparent that change in monitored object course as well as position is visible on the map. When the aircraft flew along the desired route it left marks on the map indicating a history of its antecedent positions. Additionally the precise information of aircrafts position and attitude was on an ongoing basis actualized. Test evidenced that graphical content representing pitch, roll and yaw angles, is helpful in raising the awareness of aircrafts attitude. Besides the control surface position area is useful in understanding the correlation between them and the movement of the aircraft and may be useful in future development of autopilot system. Some of parameters are displayed with two different units increasing their usefulness.

\section{CONCLUSIONS}

The presented design of ground control station allows not only monitoring current position and attitude of the aircraft through utilization of different controls, but planning the aircrafts route and adding new waypoint to it as well. The favorable side of the design lies on both, visualization and display of the monitored parameters. While the visualization gives coarse but swiftly and effortlessly understandable information, distinct controls display it in more precise way.

Having accomplished the design of ground control station it is possible to further improve its content by editing or adding new elements. It is possible to add three dimensional map which will increase the functionality of existing one by adding the information of the height (Siebert and Teizer, 2014). Furthermore 3D map gives the opportunity of visualizing modeled objects like buildings, trees and other obstacles which role is significant in the context of route planning. If the autopilot will be concerned, the possibility of adding features such as controller gains tuning window and graphs plotting window is arising. Likewise the performed ground control station may be helpful in the development process of an autopilot system for the needs of flight simulator (Cetin et al., 2011).

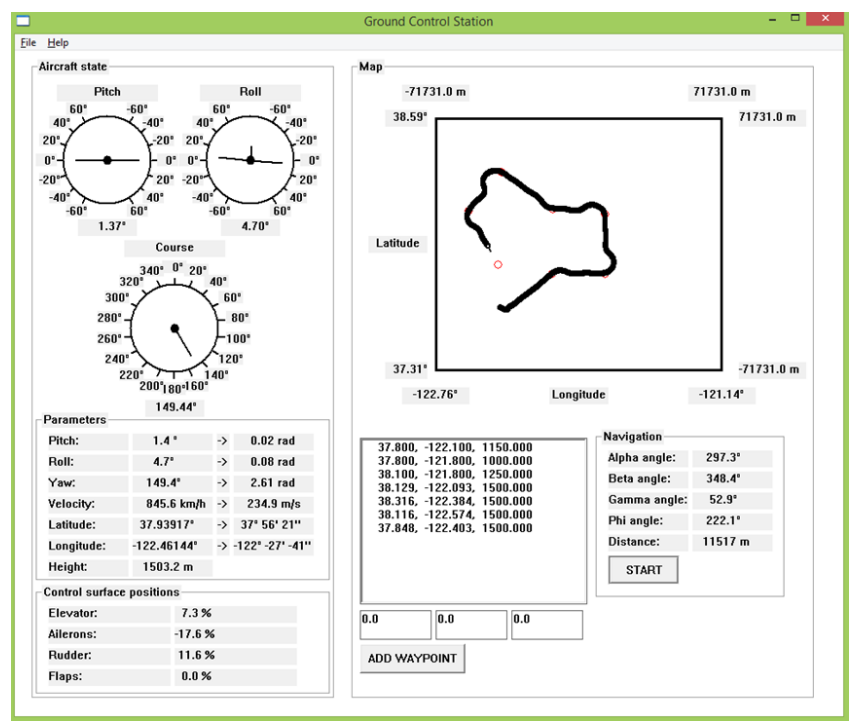

Fig. 7. Ground control station during the testing

Created GCS on the basis of flight simulator, may be further easily adopted to cooperate with real aircrafts, allowing taking control over their autopilots. This alteration may be a significant step in creating a complex unmanned aerial system consisting of aircraft, its autopilot and GCS itself. Aforementioned software may be further developed to suit specific mission requirements (Han et al., 2013). The adaptation can involve extension of the GCS functionalities to video stream handling, sensor data visualization, control over auxiliary onboard devices, cooperation with terrestrial system.

Future work may also be based on the conception of multi UAV systems, extending the capabilities of the presented ground control station for monitoring and controlling several of them (Boccalatte et al., 2013; Zheng et al., 2004; Dydek et al., 2013; Garcia et al., 2010; Sahingoz et al. 2014). The maximum number of different UAVs that can be supervised is limited by the operators workload capabilities and the design of ground control station. Systems that consist of multi unmanned aerial vehicles can hasten the mission realization, decrease the influence of timedependent factors e.g. weather and increase the success plausibility. Furthermore this approach gives the possibility of realizing tasks that can't be performed by single UAV, or assuring mission 
continuity in the case of low energy level of operating unmanned aerial vehicle (Damilano et al., 2013; Maza et al., 2010; Perez et al., 2013).

\section{REFERENCES}

1. Boccalatte M., Brogi F., Catalfamo F., Maddaluno S., Martino M., Mellano V., Rosazza P. P., Solitro F., Torasso P., Torta G. (2013), A multi-UAS cooperative mission over non-segregated civil areas, Journal of Intelligent and Robotic Systems, 70, 275-291.

2. Cetin O., Kurnaz S., Kaynak O. (2011), Fuzzy logic based approach to design of autonomous landing system for unmanned aerial vehicles, Journal of Intelligent and Robotic Systems, 61, 239-250.

3. Damilano L., Guglieri G., Quagliotti F., Sale I., Lunghi A. (2013), Ground control station embedded mission planning for UAS, Journal of Intelligent and Robotic Systems, 69, 241-256.

4. Dydek Z. T., Annaswamy A. M., Lavretsky E. (2013), Adaptive configuration control of multiple UAVs, Control Engineering Practice, 21, 1043-1052.

5. Garcia R., Barnes L. (2010), Multi-UAV simulator utilizing X-Plane, Journal of Intelligent and Robotic Systems, 57, 393-406.

6. Jinlu H., Yaojin X., Long D., YangQuan C. (2013), Low-cost multiUAV technologies for contour mapping of nuclear radiation field, Journal of Intelligent and Robotic Systems, 70, 401-410.
7. Maza I., Kondak K., Bernard M., Ollero A. (2010), Multi-UAV Cooperation and Control for Load Transportation and Deployment, Journal of Intelligent and Robotic Systems, 57, 417-449.

8. Perez D., Maza I., Caballero F., Scarlatti D., Casado E., Ollero A. (2013), A ground control station for a multi-UAV surveillance system, Journal of Intelligent and Robotic Systems, 69, 119-130.

9. Sahingoz O. K. (2014), Generation of Bezier Curve-Based Flyable Trajectories for multi-UAV systems with Parallel Genetic Algorithm, Journal of Intelligent and Robotic Systems, 74, 499-511.

10. Siebert S., Teizer J. (2014), Mobile 3D mapping for surveying earthwork projects using an Unmanned Aerial Vehicle (UAV) system, Automation in Construction, 41, 1-14.

11. Zheng C., Ding M., Zhou C., Li L. (2004), Coevolving and cooperating path planner for multiple unmanneed air vehicles, Engineering Applications of Artificial Intelligence, 17, 887-896.

12. http://msdn.microsoft.com/en-us/library

13. http://www.airware.com/

14. http://www.diamondaircraft.com/

15. http://www.flightgear.org/

16. http://www.openpilot.org/

17. http://www.qgroundcontrol.org/

18. http://www.students.mimuw.edu.pl/SO/Linux

19. http://www.tools.ietf.org/html/rfc793

Acknowledgements: The researchers are financed by Bialystok University of Technology as the own work (No. MB/WM/16/2014). 